(C) The Author(s), 2020. Published by Cambridge University Press on behalf of The Nutrition Society. This is an Open Access article, distributed under the terms of the Creative Commons Attribution licence (http://creativecommons.org/licenses/by/4.0/), which permits unrestricted re-use, distribution, and reproduction in any medium, provided the original work is properly cited.

\title{
Policy-Relevant Paper
}

\section{An overview of the Scientific Advisory Committee on Nutrition's position statement on nutrition and older adults living in the community}

\author{
Margie van Dijk ${ }^{1}$, Adrienne Cullum ${ }^{1 *}$, Gillian Swan ${ }^{1}$, Emma Peacock $^{1}$, Gill Fine ${ }^{2}$, Siân Robinson ${ }^{2}$, \\ Harry McArdle ${ }^{2}$, Angus Walls ${ }^{2}$, Stella Walsh ${ }^{2}$ on behalf of the Scientific Advisory Committee on Nutrition \\ (SACN) \\ ${ }^{1}$ Public Health England SACN Secretariat \\ ${ }^{2}$ Public Health England SACN Member
}

Key words: Older adults: Elderly: Healthy ageing: SACN: Diet: Nutrition

In January 2021, the Scientific Advisory Committee on Nutrition (SACN) published a position statement on nutrition and older adults living in the community ${ }^{(1)}$. The position statement provides an overview of the currently available evidence on nutrition in older adults and its impact on healthy ageing. While it is not intended to be a comprehensive review of the evidence base, it does draw conclusions on the current evidence and makes research recommendations.

Although SACN reports have reviewed recommendations for older adults, including for energy and vitamin $\mathrm{D}^{(2,3)}$, nutrition and older adults were last considered by the Committee on Medical Aspects of Food and Nutrition Policy (COMA) as a dedicated topic in $1992^{(4)}$. Since then, the age structure of the population has undergone considerable change with growth in the proportion and number of older adults. In this context, SACN agreed to review the more recent evidence base and make recommendations where possible.

In 2018, SACN convened a working group to consider the evidence on dietary intakes and patterns of older adults compared with current UK recommendations and key nutritional issues relevant to age-related health outcomes. The working group agreed to look at evidence in different age groups of older adults (in particular 65-74 years and 75 years and above) where available. The statement also presents, for the first time, energy and nutrient intakes for older adults aged 80 years and over from the National Diet and Nutrition Survey (NDNS).

SACN's remit is to assess the risks and benefits of nutrients, dietary patterns, food or food components to health by evaluating scientific evidence and to make dietary recommendations for the UK based on its assessment ${ }^{(5)}$. SACN has a public health focus and only considers evidence in healthy populations unless specifically requested to do otherwise. This means that while frailty and malnutrition are important considerations in older adults, evidence relating to older adults who are frail, malnourished and/or living in residential or nursing care was out of scope for this position statement as was evidence related to older adults with chronic conditions such as cancer or type 2 diabetes or the treatment or management of conditions.

\section{Background}

The demographics of the UK population have changed in recent decades with an increased number and proportion of older adults. In 1998, about 1 in 6 people (15.9\%) were aged 65 years and over; by 2018, this figure had increased to 1 in 5 people (18.3\%) with projections to reach about 1 in 4 people $(24.2 \%)$ by $2038^{(6)}$

Ageing is a life-long process. How people age, and their health in later life, can be modified by a range of influences throughout life. A broad range of cultural, environmental and social factors can impact on health and dietary intake in older adulthood. The risk of many diseases and chronic health conditions increases with age, and more than half of UK adults aged over 65 years have at least two chronic health conditions that may affect day to day living.

Nutrition, age-related physiological changes and health are often interconnected. In some cases, age-related physiological

Abbreviations: NDNS, National Diet and Nutrition Survey; PCS, prospective cohort study; RCT, randomised controlled trial; SACN, Scientific Advisory Committee on Nutrition.

* Corresponding author: Adrienne Cullum, email Adrienne.Cullum@phe.gov.uk 
changes can impact on nutritional intake and status; in others, nutritional intake and status can impact on how people age.

\section{Current UK Government Dietary Recommendations}

The position statement reviewed the dietary intake evidence and compared it with the dietary recommendations for older adults in the UK. Dietary reference values for energy, macronutrients and micronutrients are set by SACN and its predecessor $\mathrm{COMA}^{(7)}$. Estimated average requirements for energy are set for males and females in 10-year age bands including 65-74 years and 75 years and over. Dietary reference values for protein are set as reference nutrient intakes for males and females aged over 50 years based on a single reference nutrient intake per kg body weight across all age groups. For fats, carbohydrates, fibre and salt, dietary reference values are set for adults aged 19 years and over as a single group. For micronutrients, the dietary reference values are generally the same for all adult age groups with the exception of thiamin and niacin (based on energy intake), vitamin $\mathrm{B}_{6}$ (based on protein intake) and Fe.

\section{Dietary intakes and nutritional status}

Overall, data from the NDNS ${ }^{(8)}$ indicate that older adults exceed maximum recommendations for intakes of saturated fat, free sugars and salt and fail to meet recommendations for fruit and vegetables, fibre and oily fish. This is similar to the dietary intakes for the wider UK adult population.

\section{Energy and $\mathrm{BMI}$}

NDNS data indicate that energy intakes decline with age with mean energy intakes below estimated average requirements in older adults as well as in younger age groups. In parallel, NDNS data on BMI also indicate high levels of overweight and obesity in older adults: $87 \%$ of men and $68 \%$ of women aged $65-74$ years, and $69 \%$ of men and $58 \%$ of women aged 75 years and over were living with overweight or obesity. For 65-74 year olds, this was a higher percentage than for younger adults (aged 19-64 years). In comparison, the prevalence of underweight was low; $<1 \%$ of men and $3 \%$ of women aged $65-74$ years, and $7 \%$ of men and $3 \%$ of women aged 75 years and over were underweight.

These figures broadly reflect data from the Health Survey for England ${ }^{(9)}$, which is based on a much larger sample size. The high prevalence of overweight and obesity in older adults suggests that energy intakes are likely to be adequate in this age group at a population level and, as for younger adults, low reported intakes are at least in part due to underreporting.

\section{Macronutrients}

Mean protein intakes met the reference nutrient intake in all age/sex groups, although they also showed a decline with age from 19-64 years through to the 75 years and over age groups. Mean intakes of both saturated fat and free sugars exceeded the maximum recommendations. Intakes of saturated fat and, for men, free sugars were higher in adults aged 75 years and over than in the 65-74 year age group. Mean fibre intakes were below recommendations in both age groups, as was the case for younger adults, and were lower in the 75 years and over age group than in those aged 65-74 years.

\section{Micronutrients}

There was some evidence of low micronutrient intakes, particularly in women and in the 75 years and over age group (e.g. for women aged 75 years and over: vitamin A, riboflavin and folate, $\mathrm{Fe}, \mathrm{Ca}$ and $\mathrm{Zn}$ ). There was also evidence of poor vitamin $\mathrm{D}$ and folate status and to a lesser extent poor Fe and vitamin $\mathrm{B}_{12}$ status.

In summary, the diets of the 65-74 years age group are similar to, and for some foods and nutrients, better than younger adults, particularly for women, as explained above. However, for the 75 years and over age group, there was evidence of a decline in energy, protein and micronutrient intakes, particularly in women. Mean protein intakes did meet the reference nutrient intake in all age/sex groups.

Based on limited data for the 80 years and over age group, there was no evidence of poorer diets in this age group compared with the 75 years and over age group. However, it is important to note that data on this age group were available only for energy and nutrient intakes and consumption of selected foods and were collected over a longer time period than the data for younger age groups.

An important consideration in interpreting the NDNS data is that although the NDNS sample is designed to be representative of the population in each age group, it is possible that the sample may under-represent people who have long-term health conditions which makes it more difficult to participate in a survey.

\section{Methods}

Public Health England conducted a search of key online databases (Embase, MEDLINE, Cochrane Library and Food Science Technology Abstracts) in November 2018 to identify relevant English language articles published between 1990 and 9 November 2018, using an agreed list of search terms focusing on nutrition and healthy ageing. There was particular interest in the following outcomes: mortality, musculoskeletal health, cardiovascular health, cancer, immune function, oral health, weight change, quality of life and eye health.

The position statement included only evidence from systematic reviews and meta-analyses, primarily of randomised controlled trials (RCT) and prospective cohort studies (PCS), reflecting SACN's framework for the evaluation of evidence ${ }^{(5)}$. Systematic reviews that included case-control or cross-sectional studies alongside RCT and PCS were also included, although the results are given less priority. Further inclusion criteria were adults aged 65 years and over (or mean age of $\geq 60$ years) including relevant sub-group analyses and free-living, predominantly healthy populations. SACN invited interested parties to highlight relevant evidence which satisfied the inclusion criteria for the position statement through a call for evidence between 9 January 2019 and 5 February 2019.

The quality of included systematic reviews and meta-analyses was assessed by the SACN Framework for the Evaluation of Evidence $^{(5)}$ and the AMSTAR 2 tool $^{(10)}$. The latter was used 
to assess the methodological quality of included systematic reviews, and to aid discussion, but it was not used to draw conclusions on the evidence.

\section{Evidence identified}

Thirty systematic reviews, fifteen with meta-analyses, were included in the position statement. The majority of the identified systematic reviews focused on musculoskeletal health outcomes, including sarcopenia (muscle strength, muscle mass, physical performance), frailty and bone health. No evidence that met the inclusion criteria was identified on oral health, gastrointestinal tract health, eye health, hydration or skin and wound health.

\section{Musculoskeletal health}

It was not possible to draw conclusions on the association between BMI and musculoskeletal health as evidence from systematic reviews reported on different musculoskeletal health outcomes and most evidence was from cross-sectional studies.

Evidence from meta-analyses of RCT suggested no significant effect of protein supplements on measures of musculoskeletal health in older adults. Evidence from systematic reviews of observational studies on the association between dietary protein intake and musculoskeletal health was unclear. However, different measures of musculoskeletal health were considered in the various systematic reviews, and the studies had a number of limitations (discussed below).

As SACN published its report on Vitamin D and Health in $2016^{(2)}$, only systematic reviews published after the search periods covered in the Vitamin D and Health report were included. The four identified systematic reviews did not provide enough evidence to change the conclusions of the 2016 SACN report on Vitamin D and Health.

There was very little evidence identified from RCT on dietary patterns and musculoskeletal health outcomes. Evidence from observational studies suggested that adherence to 'healthy' diets or Mediterranean dietary patterns improved measures of musculoskeletal health. However, different definitions of a 'healthy' diet were used, and much of the evidence was from cross-sectional studies. Overall, the evidence was too mixed to draw conclusions.

\section{CVD}

There was mixed evidence from one systematic review that compliance with a Mediterranean dietary pattern reduced the risk of CVD in older adults.

No systematic reviews were identified which looked specifically at the relationship between dietary fats and cardiovascular health outcomes in older adults. This aligns with the SACN review on Saturated Fats and Health ${ }^{(11)}$ which concluded that there was limited evidence on saturated fats and health in older adults and that this evidence did not provide a basis for changing existing recommendations for this age group ${ }^{(11)}$.

\section{Cancer}

Evidence that was available from one systematic review of PCS on Mediterranean dietary patterns and cancer broadly reflected the wider evidence base for the general adult population, that 'healthier' dietary patterns may reduce the risk of some cancers.

\section{Other outcomes}

As SACN published its Statement on Diet, Cognitive Impairment and Dementias in $2018^{(12)}$, nutrition and cognition were not considered in this position statement. The 2018 position statement concluded that adherence to Mediterranean dietary patterns was associated with a reduced risk of mild cognitive impairment and dementias, including Alzheimer's disease. There was no evidence of protective effects for any of the individual nutrients thought to account for the health benefits of Mediterranean dietary patterns ${ }^{(12)}$.

For the outcomes of overall mortality and morbidity, immune function, weight change and quality of life, there were gaps in the evidence and a limited number of systematic reviews specifically studying these outcomes in adults aged 65 years and over.

\section{Issues identified in the evidence base}

The position statement set out to identify evidence from systematic reviews on nutrition and age-related health outcomes of community-dwelling older adults. However, there was a lack of evidence identified in this age group for most outcomes, in particular, in relation to major health outcomes, such as CVD and cancers. There was insufficient evidence to draw any conclusions for older adults in black, Asian and minority ethnic groups, as most of the systematic reviews or meta-analyses did not consider ethnicity. Given the demographic shifts in the population, SACN recommended that there is a need for more research in this age group from high-quality RCT and longitudinal studies.

Understanding the evidence from older populations poses a number of challenges, which need to be considered for future research. This includes the following:

- Older adults are a heterogeneous group, and chronological age is a poor indicator of an older adult's health status, which may differ widely. Some older adults are healthy and active, while others have multiple conditions, diseases or disabilities.

- There can be significant differences between the youngest old and oldest old in terms of their physiological ability to respond to ill health (e.g. infections or chronic disease).

- Dietary assessments in some older people may also pose a number of challenges and ethical considerations in relation to a participant's cognitive and/or physical ability, the high burden imposed on study participants and possible indirect reporting of food intakes by a carer or spouse. These factors can all impact the accuracy of assessment.

- Community-dwelling older adults are heterogeneous in terms of the support they receive with some living independently and others receiving support from professional or familymember carers.

- Intervention studies in this age group tend to consider specific nutrients rather than broader dietary interventions.

The position statement identified a number of specific limitations in the evidence base. Some of the systematic reviews only 
included small numbers of RCT or PCS, which were often short in duration and included a small number of participants. Some of the systematic reviews included case-control and crosssectional studies alongside PCS and did not always present results for these separately. There was heterogeneity in the definition of healthy ageing between publications and the participants included; some reviews excluded primary studies that considered participants with existing chronic conditions, while others included them. Some of the systematic reviews and/or meta-analyses included a number of studies conducted in hospital settings or institutions. The age range of participants varied between studies, making the comparison of results difficult. For example, some studies included adults older than 50 years, while others included adults from age 60 or 65 years. In protein supplementation studies, the type and source of protein differed between studies and some studies did not provide information on the protein type and source. In dietary pattern studies, the definition and assessment of a Mediterranean dietary pattern differed between studies. Dietary assessment methods differed between studies making comparisons difficult. Studies often did not take into account mediating factors that are common in older age, such as swallowing or poor dentition.

It is important to note that although the review explored a wide range of health outcomes relevant to older adults, there are a number of outcomes that were not considered in the review, including mental health, and little or no evidence was identified from systematic reviews for some of the outcomes including overall mortality and morbidity, immune function, weight change and quality of life.

\section{Conclusions}

The SACN position statement on nutrition and older adults living in the community is based on a robust evaluation of the evidence from systematic reviews. It highlights a lack of high-quality evidence in this population group, in particular for major health outcomes such as CVD and cancer, and also in ethnic minority groups. The position statement identified a range of research recommendations, including the need for high-quality evidence from RCT and longitudinal studies on nutrition-related outcomes for older adults living in the community.

Based on the present review of the available literature, the position statement concludes that there is not enough highquality evidence for SACN to consider conducting a full risk assessment on older adults at this time. SACN will continue to consider older adults as a sub-group within relevant future risk assessments.

\section{Acknowledgements}

The position statement was developed by the SACN older adults working group and reviewed and approved by the full SACN committee. A list of working group and SACN members is available here: https://www.gov.uk/government/groups/scientificadvisory-committee-on-nutrition.

We would also like to thank Professor Ann Prentice from the Medical Research Council (MRC) Nutrition and Bone Health
Research Group, Cambridge, who was SACN Chair during the development of the report until May 2020.

SACN is an independent committee of Public Health England. The position statement was funded by Public Health England.

M. v. D. prepared the first draft of the manuscript, which was revised by A. C., G. S., G. F. and S. R. All authors read and approved the final manuscript.

The authors declared that there are no conflicts of interest.

\section{References}

1. Scientific Advisory Committee on Nutrition (SACN) (2020) Position Statement on Nutrition and Older Adults Living in the Community. https://www.gov.uk/government/collections/sacnreports-and-position-statements\#position-statements (accessed November 2020).

2. Scientific Advisory Committee on Nutrition (SACN) (2016) Vitamin D and health. https://www.gov.uk/government/ publications/sacn-vitamin-d-and-health-report (accessed November 2020).

3. Scientific Advisory Committee on Nutrition (SACN) (2012) Dietary reference values for energy. https:/Www.gov.uk/ government/publications/sacn-dietary-reference-values-for-energy (accessed November 2020).

4. Department of Health (1992) Report on Health and Social Subjects. No. 43. Report of the Working Group on the Nutrition of Elderly People of the Committee on Medical Aspects of Food Policy. London: HMSO. https://www.gov.uk/government/ publications/coma-reports (accessed November 2020).

5. Scientific Advisory Committee on Nutrition (SACN) (2020) SACN Framework for the Evaluation of Evidence. https:// www.gov.uk/government/groups/scientific-advisory-committeeon-nutrition\#framework-for-the-evaluation-of-evidence (accessed November 2020).

6. Office for National Statistics (2019) Overview of the UK population: August 2019. https://www.ons.gov.uk/peoplepopulationan dcommunity/populationandmigration/populationestimates/ articles/overviewoftheukpopulation/august2019 (accessed November 2020).

7. Department of Health (1991) Report on Health and Social Subjects no. 41. Report of the Panel on Dietary Reference Values of the Committee on Medical Aspects of Food Policy. Dietary Reference Values for Food Energy and Nutrients for the United Kingdom. London: HMSO.

8. Roberts C, Steer T, Maplethorpe N, et al. (2018) National Diet and Nutrition Survey, Results from Years 7 and 8 (combined) of the Rolling Programme (2014/2015 to 2015/2016). https:// www.gov.uk/government/statistics/ndns-results-from-years7-and-8-combined (accessed November 2020).

9. NHS Digital (2018) Health Survey for England 2017. https:// digital.nhs.uk/data-and-information/publications/statistical/ health-survey-for-england/2017 (accessed November 2020).

10. Shea BJ, Reeves BC, Wells G, et al. (2017) AMSTAR 2: a critical appraisal tool for systematic reviews that include randomised or non-randomised studies of healthcare interventions, or both. BMJ 358, j4008.

11. Scientific Advisory Committee on Nutrition (SACN) (2019) Saturated Fats and Health. https://www.gov.uk/government/ publications/saturated-fats-and-health-sacn-report (accessed November 2020)

12. Scientific Advisory Committee on Nutrition (SACN) (2018) Diet, Cognitive Impairment and Dementias. https://www.gov.uk/ government/publications/sacn-statement-on-diet-cognitiveimpairment-and-dementia (accessed November 2020). 